\title{
How many Canadian health workers remain unvaccinated?
}

\author{
n Cite as: CMAJ 2021 August 16;193:E1259-60. doi: 10.1503/cmaj.1095956
}

Posted on cmajnews.com on July 29, 2021

everal European countries and major health networks in the United States recently mandated SARS-CoV-2 vaccinations for health workers, prompting calls for similar policies in Canada.

While most Canadian health workers took advantage of early access to SARSCoV-2 vaccines, more than six months later uptake has slowed, with about one in 10 health workers remaining unvaccinated in some provinces.

Quebec's ministry of health and social services told CMAJ that $89 \%$ of public and private health workers in the province have received at least one dose of a SARS-CoV-2 vaccine. According to a spokesperson, the ministry is "doing everything we can" to increase uptake, including requiring health workers to disclose their vaccination status to employers and undergo testing three times a week if they're not immunized.

A spokesperson for Manitoba estimated that more than $80 \%$ of the province's 62000 health workers have received at least one dose of a SARS-CoV-2 vaccine. Manitoba recently mandated staff at personal care homes to show proof of vaccination to work at multiple sites. The province also runs walk-in vaccination clinics and allows workers to take a three-hour paid leave to receive the shots.

Details on the number of health workers vaccinated in other provinces are less clear.

As of mid-July, nearly one in 10 longterm care workers in Ontario had not received a first SARS-CoV-2 vaccine. The province has since mandated long-term care workers to report their vaccination status to employers - unvaccinated staff without medical exemptions must undertake training on the importance of immunization.

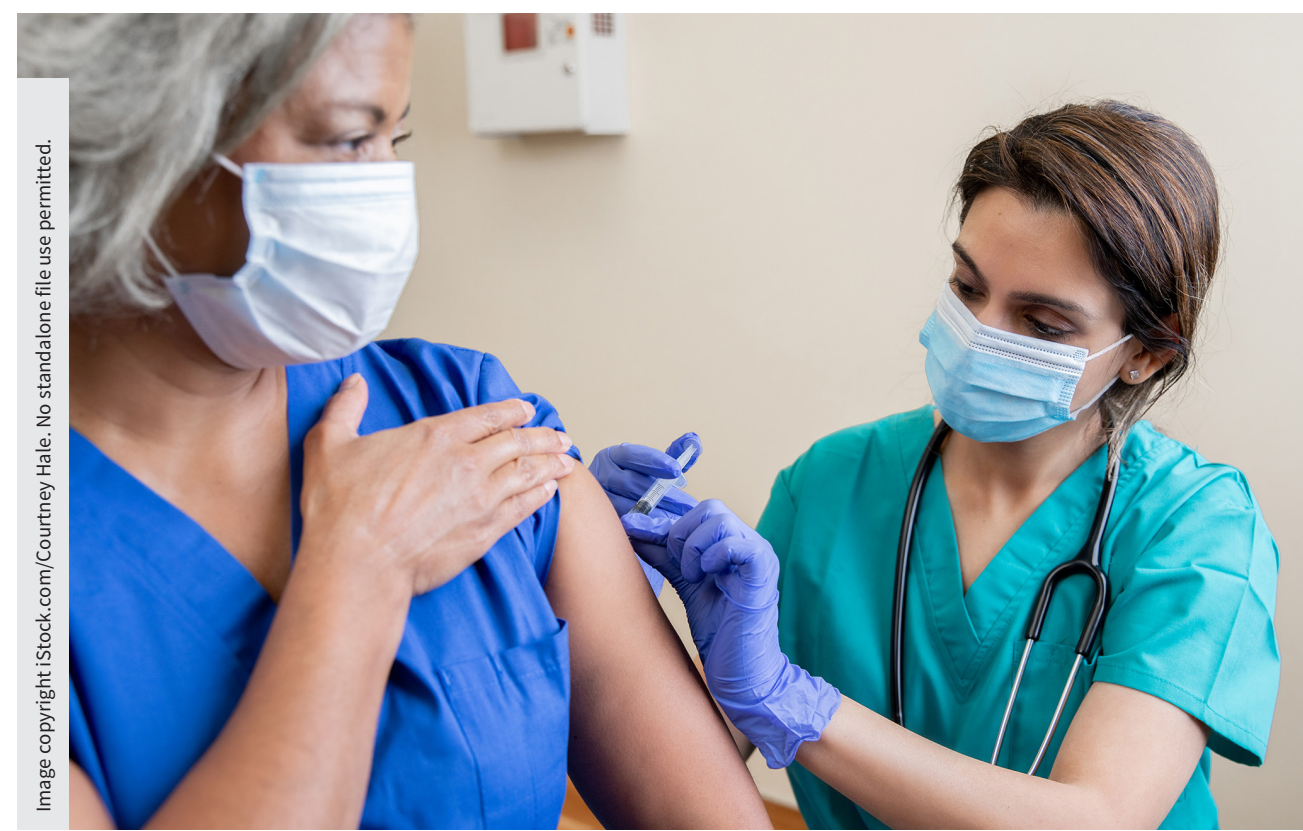

Calls to mandate SARS-CoV-2 vaccination for health workers underscore hesitancy and access issues.

British Columbia has a similar policy and requires unvaccinated long-term care workers to wear masks and undergo testing three times a week. Anecdotally, vaccination rates among long-term care staff lag those of residents, but officials haven't disclosed exact numbers.

Some 35000 self-identified health workers in Newfoundland and Labrador have received their first SARS-CoV-2 vaccine. It's unclear what percentage of the total health workforce that number represents because the province doesn't keep records on private health providers. Still, according to a spokesperson, "there are no indications that there is a hesitancy among health care workers to get the vaccines."

According to Alberta Health Services, the "vast majority" of its staff and physicians are immunized. A spokesperson said the health authority is running an awareness campaign for those who still have questions about the vaccines, noting, "we know that different people have different feelings and concerns about COVID-19, and that's okay."

Nova Scotia isn't tracking health workers' vaccine uptake and doesn't require them to report their vaccination status to employers. Generally, the province is aiming to vaccinate $75 \%$ of all residents by the end of August.

The Yukon isn't tracking health workers' vaccination status either, although a spokesperson said the government "strongly" encourages all employees to get vaccinated. "For health care workers, we have had focused educational initiatives for our employees regarding the vaccine, along with offering vaccinations at work." 
Other provinces and territories did not immediately respond to requests for information.

Many Canadian physicians and nurses, and some health care associations, are urging officials to require SARS-CoV-2 vaccination as a condition of employment in hospitals, long-term care, and other health care settings. They argue that health workers have a duty to avoid spreading the virus to vulnerable patients and point out that vaccinations against other diseases like measles are already mandatory.

The emergence of more transmissible and dangerous variants of SARS-CoV-2 has added urgency to the appeal. So far, more than $80 \%$ of eligible Canadian residents have had at least one SARS-CoV-2 shot, and more than $60 \%$ are fully vaccinated. But, without approved SARSCoV-2 vaccines for children under 12 , adults will have to pick up the slack to achieve the $75 \%-90 \%$ coverage required for herd immunity.

\section{Vaccine hesitancy still a hurdle}

While published research on Canadian health workers' uptake of SARS-CoV-2 vaccines is limited, studies suggest that vaccine hesitancy isn't uncommon, especially among nonphysicians.

At the start of Canada's SARS-CoV-2 vaccine rollout, nearly one-third of Quebec nurses said they were reluctant to accept vaccination, while one in 10 said they probably or definitely would avoid it. And although Ontario physicians reported higher than average intention to vaccinate at that time, nonphysician health workers were about as likely as the general public to say they would get the jabs (80\%).

In other countries, health workers' acceptance of SARS-CoV-2 vaccines has ranged from roughly $28 \%$ to $82 \%$. Acceptance was lower among women, Black and Latinx, and rural and politically conservative health workers.

Health workers' overall attitudes toward vaccination were positive, according to the Evidence Synthesis Network COVID-19 Collaboration in Practice. Still, concerns about the speed of development and effectiveness of SARS-CoV-2 vaccines were prevalent. The group noted that experiences of racial discrimination also factored in some health workers' hesitancy.

Several months into the United States' vaccine rollout, one in four American hospital workers had not received their first shot, according to an analysis of data collected by the Department of Health and Human Services. In the 50 largest hospitals, one in three workers was unvaccinated.

Anecdotal reports suggest that health workers are not immune to COVID-19 conspiracy theories and misinformation spreading in the general population. The proliferation of misinformation in nurses' groups lately spurred the Canadian Nurses Association and other organizations to issue social media guidelines to discourage nurses from spreading fringe theories.

According to Tim Guest, president of the Canadian Nurses Association, "as trusted members of the public, we need to be cognizant of our unique position to share information."

However, vaccine hesitancy isn't the only barrier to health workers getting vaccinated. Some note that cultural and scheduling issues may pose obstacles too.

"If you're talking about physicians, that's one thing. But if you're actually looking at the fuller spectrum of health care workers, it's a different situation," said Sabina Vohra-Miller, cofounder of the South Asian Health Network in Toronto. For example, personal support workers, long-term care workers and custodial staff may work multiple jobs and long hours to make ends meet. "You don't necessarily have people coming from as much privilege or accessibility."

Research on interventions to boost heath workers' uptake of SARS-CoV-2 vaccines is lacking, given how recently Canada's rollout started. One preprint study of nonphysician health workers linked hesitancy with safety concerns and mistaken beliefs that the vaccine wasn't necessary if you're healthy. The authors concluded that campaigns targeting these health workers should "leverage emotion to capture interest while reinforcing the safety and low risk of side effects."

According to the Evidence Synthesis Network, the most successful vaccination drives targeting health workers before the COVID-19 pandemic have been multifaceted, involving easy access to vaccines, educational efforts, reminders and incentives, organizational changes, and mandatory vaccination policies in some cases.

Outside the context of a global crisis, though, campaigns promoting flu and other nonmandatory vaccines "solely based on education and promotion, or on-site vaccination, did not regularly exceed an absolute vaccination coverage of $40 \%$."

\section{Lauren Vogel and Diana Duong, CMAJ}

Content licence: This is an Open Access article distributed in accordance with the terms of the Creative Commons Attribution (CC BY-NC-ND 4.0) licence, which permits use, distribution and reproduction in any medium, provided that the original publication is properly cited, the use is noncommercial (i.e., research or educational use), and no modifications or adaptations are made. See: https://creativecommons.org/ licenses/by-nc-nd/4.0/ 